\title{
Pre-breakdown and Breakdown Mechanisms of an Inhibited Gas to Liquid Hydrocarbon Transformer Oil under Negative Lightning Impulse Voltage
}

DOI:

10.1109/TDEI.2017.006416

\section{Document Version}

Accepted author manuscript

Link to publication record in Manchester Research Explorer

Citation for published version (APA):

Lu, W., Liu, Q., \& Wang, Z. D. (2017). Pre-breakdown and Breakdown Mechanisms of an Inhibited Gas to Liquid Hydrocarbon Transformer Oil under Negative Lightning Impulse Voltage. IEEE Transactions on Dielectrics and Electrical Insulation, 24(5), 2809-2818. https://doi.org/10.1109/TDEI.2017.006416

Published in:

IEEE Transactions on Dielectrics and Electrical Insulation

\section{Citing this paper}

Please note that where the full-text provided on Manchester Research Explorer is the Author Accepted Manuscript or Proof version this may differ from the final Published version. If citing, it is advised that you check and use the publisher's definitive version.

\section{General rights}

Copyright and moral rights for the publications made accessible in the Research Explorer are retained by the authors and/or other copyright owners and it is a condition of accessing publications that users recognise and abide by the legal requirements associated with these rights.

\section{Takedown policy}

If you believe that this document breaches copyright please refer to the University of Manchester's Takedown Procedures [http://man.ac.uk/04Y6Bo] or contact uml.scholarlycommunications@manchester.ac.uk providing relevant details, so we can investigate your claim.

\section{OPEN ACCESS}




\title{
Pre-breakdown and Breakdown Mechanisms of an Inhibited Gas to Liquid Hydrocarbon Transformer Oil under Negative Lightning Impulse Voltage
}

\author{
W. Lu, Q. Liu and Z.D. Wang \\ School of Electrical and Electronic Engineering \\ The University of Manchester \\ Manchester M13 9PL, UK
}

\begin{abstract}
In this paper, streamer and breakdown phenomena and their mechanisms of an inhibited Gas-To-Liquid (GTL) transformer oil under standard negative lightning impulse voltages were studied. A conventional inhibited mineral oil was also tested as the benchmark. Experiments were carried out in $25 \mathrm{~mm}$ and $50 \mathrm{~mm}$ point-plane gaps. Streamer and breakdown phenomena of both oils were observed from the streamer inception voltage level up to the voltage level at which fast streamer appears with velocity over $10 \mathrm{~km} / \mathrm{s}$. The results showed that the GTL oil has a higher breakdown voltage and a higher acceleration voltage compared to the mineral oil. This is due to the fact that the GTL oil has a much lower aromatic content compared to the mineral oil. The streamer investigations showed that the development of fast streamers in both oils is the consequence of transition from dark streamer channels to bright streamer channels. By comparing with the results obtained under positive polarity [1], the differences in the propagation mechanisms between the fast negative streamers and fast positive streamers were explained.
\end{abstract}

Index Terms - Streamer, gas-to-liquid (GTL), mineral oil, lightning impulse, breakdown, transformer.

\section{INTRODUCTION}

IN a previously published paper, the streamer and breakdown mechanisms of a new Gas-to-Liquid (GTL) transformer oil were studied under positive standard lightning impulse voltages [1]. The results showed that under positive polarity, the GTL oil has a higher breakdown voltage but a lower acceleration voltage compared to conventional mineral oil. This is due to the fact that the GTL oil has a much lower aromatic content compared to the mineral oil [1]. Based on detailed characterizations of the transition process from dark streamer channels to bright streamer channels, a model was proposed where a charge splitting process is considered to be responsible for the propagation of dark channels and a charge amplification process responsible for the propagation of bright channels [1].

In past decades, pre-breakdown streamer phenomena in liquids under negative impulse voltages were thoroughly investigated, considering the effects of different testing conditions such as voltage waveform, liquid nature, additives and hydrostatic pressure [2-14]. Concerning the detailed propagation process of fast streamers above acceleration voltage, much fewer experiments were found under negative impulse voltages than those under positive impulse voltages

Manuscript received on October 2016, in final form XX Month 2017.
[10-15]. Results in $[12,13]$ showed that under $0.5 / 1700 \mu \mathrm{s}$ step impulses, the propagation of fast negative streamers above acceleration voltage in a mineral oil consists of multiple stages. At different stages, the negative streamer propagates either as fast bright channels or as relatively slow dark channels. The presence of both reduced pressure and electron scavenging additives accelerates the propagation of fast negative streamers in mineral oil $[12,13]$. Up to now, there has not been publication describing the phenomena of negative streamers in GTL oil under impulse voltages.

This paper focuses on streamer and breakdown mechanisms of a GTL oil under negative standard lightning impulse voltages. A conventional mineral oil was also tested as the benchmark. Point-plane electrode configuration with gap distances of $25 \mathrm{~mm}$ and $50 \mathrm{~mm}$ were used. Streamer and breakdown of both oils from the initiation voltage to overstressed voltages above the acceleration voltage were analysed in terms of stopping length, velocity, area and propagation mode. The differences in streamer and breakdown phenomena between the GTL oil and the mineral oil were described. In addition, by comparing the streamer characteristics of fast streamers between positive and negative polarities, the differences in the propagation mechanisms of fast streamers were discussed in this paper. 


\section{EXPERIMENTAL DESCRIPTIONS}

A fully inhibited mineral oil Diala S3 ZX-I and a GTL based synthetic hydrocarbon oil Diala S4 ZX-I were investigated in this paper. The basic properties of these two oils were described in the previous publications [1, 16-19].

The test setup used in this paper is the same as the one used under positive polarity [1]. The experiments were performed in $25 \mathrm{~mm}$ and $50 \mathrm{~mm}$ point-plane electrode gaps under negative standard lightning impulse voltages 1.2 $( \pm 30 \%) / 50( \pm 20 \%) \mu \mathrm{s}$.

A set of 10 breakdowns by using rising voltage method (1 shot/step) following IEC 60897 [20] were carried out for each oil at each gap distance. Shadowgraph images of streamer channels were captured by using a multi-frame high-speed camera Specialised Imaging SIM-16. These streamer images obtained are 2-D shadow projection of streamer channels. Below the breakdown voltage, five streamer images were obtained at each voltage level. Above the breakdown voltage, three streamer images were obtained at each voltage level. More detailed procedures for streamer recording and breakdown tests were described in [1].

\section{STREAMER CHARACTERISTICS AND BREAKDOWN VOLTAGES}

\subsection{BREAKDOWN VOLTAGE}

Figure 1 shows the lightning impulse breakdown voltages of the GTL oil and the mineral oil measured at $25 \mathrm{~mm}$ and $50 \mathrm{~mm}$ gaps. Weibull distribution was used to fit the breakdown results to obtain the breakdown voltages at $50 \%$ breakdown probability, as given in At the same gap distance, the negative breakdown voltage of the GTL oil is higher than that of the mineral oil. It is also noticed that the difference in the breakdown voltage between the two testing oils does not change obviously with the increase of gap distance. i.e. the breakdown voltage of the mineral oil is approximately $78.5 \%$ of that of the GTL oil at both $25 \mathrm{~mm}$ and $50 \mathrm{~mm}$ gaps.

Table 1. In the following discussions of this paper, $50 \%$ probability breakdown voltage is defined as the breakdown voltage $\mathrm{V}_{\mathrm{b}}$ of the testing oils.

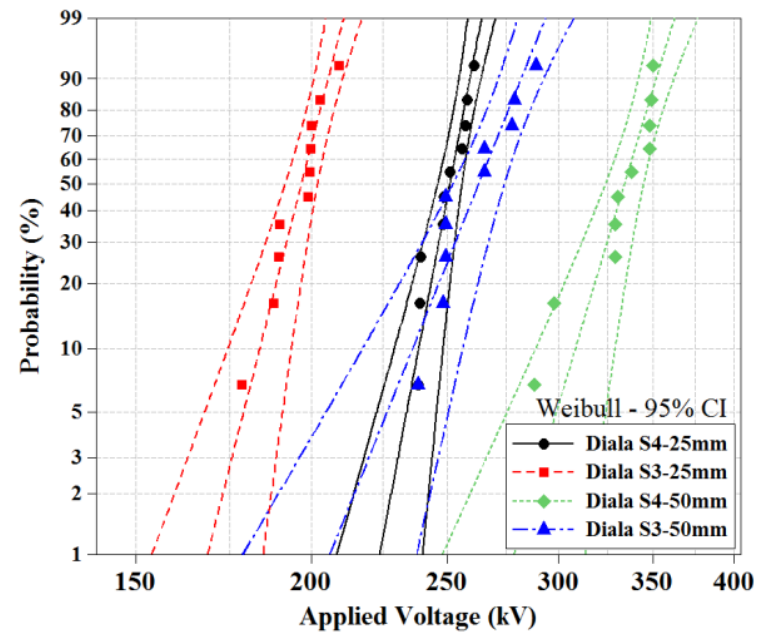

Figure 1. Cumulative probability versus breakdown voltage using Weibull fitting, $\mathrm{d}=25 \mathrm{~mm}$ and $50 \mathrm{~mm}, 95 \%$ CI stands for $95 \%$ confidence interval.

At the same gap distance, the negative breakdown voltage of the GTL oil is higher than that of the mineral oil. It is also noticed that the difference in the breakdown voltage between the two testing oils does not change obviously with the increase of gap distance. i.e. the breakdown voltage of the mineral oil is approximately $78.5 \%$ of that of the GTL oil at both $25 \mathrm{~mm}$ and $50 \mathrm{~mm}$ gaps.

TABLE 1. Weibull distribution parameters of the GTL oil and the mineral oil at $25 \mathrm{~mm}$ and $50 \mathrm{~mm}$ point-plane gaps; $\alpha$ stands for the scale parameter and $\beta$ stands for the shape parameter.

\begin{tabular}{lcccccc}
\hline & \multicolumn{5}{c}{ Weibull Parameters } \\
\cline { 2 - 7 } Type of Oil & \multicolumn{3}{c}{$\mathbf{2 5} \mathbf{~ m m}$} & \multicolumn{4}{c}{$\mathbf{5 0} \mathbf{~ m m}$} \\
\cline { 2 - 7 } Diala S4 ZX-I & 253.7 & 36.7 & 251.2 & 339.7 & 23.3 & 334.4 \\
Diala S3 ZX-I & 199.6 & 27.4 & 197.0 & 268.9 & 17.3 & 263.3 \\
\hline
\end{tabular}

\subsection{STOPPING LENGTH}

Figure 2 shows the stopping length of non-breakdown streamers in the GTL oil and the mineral oil measured at $25 \mathrm{~mm}$ and $50 \mathrm{~mm}$ gaps. The $50 \%$ breakdown voltages are presented as references. The streamer inception voltages of the testing oils were determined by the appearance of a streamer captured by the high speed camera.

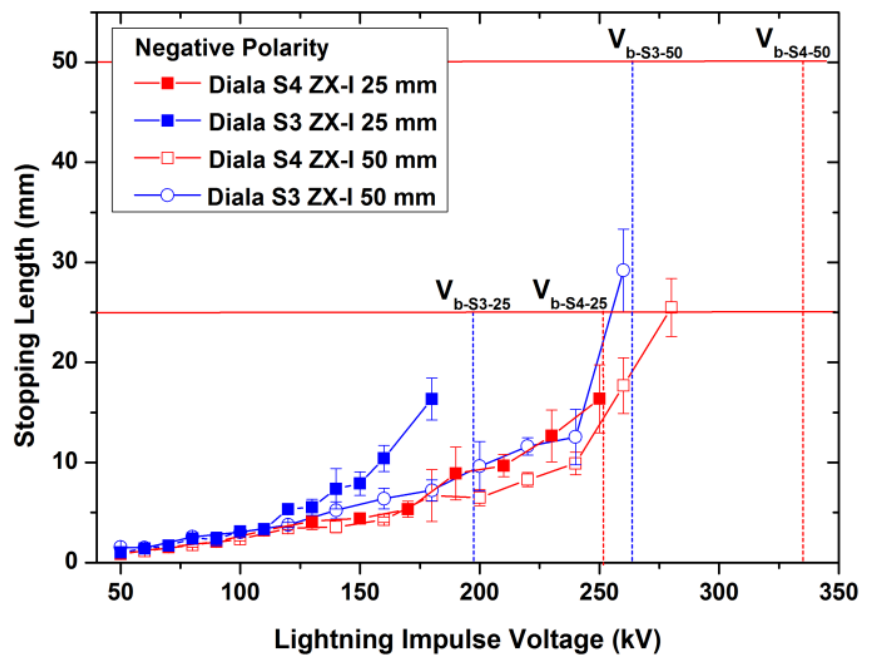

Figure 2. Streamer stopping length of the GTL oil and the mineral oil, negative polarity $\left(\mathrm{V}_{\mathrm{b}-\mathrm{S} 4-\mathrm{xx}}\right.$ and $\mathrm{V}_{\mathrm{b}-\mathrm{S} 3-\mathrm{xx}}$ stand for $50 \%$ breakdown voltages of Diala S4 ZX-I and Diala S3 ZX-I at gap distance of Xx mm, respectively).

As shown in Figure 2, at both $25 \mathrm{~mm}$ and $50 \mathrm{~mm}$ gaps, the inception voltage of negative streamer in the GTL oil, i.e. about $50 \mathrm{kV}$, is the same as that of the mineral oil. The inception voltages of negative streamers are comparable to those of positive streamers presented in the same oil [1]. However it is noticed that the shortest negative streamer captured is about $1 \mathrm{~mm}$ long, much shorter than the shortest positive streamers captured which is $7 \sim 10 \mathrm{~mm}$ long. Under negative polarity, before reaching a certain voltage level, stopping length of negative streamers in the GTL oil and the mineral oil increases slowly with applied voltage. There is no obvious difference in the increasing rate of negative streamers between the two oils. Above a certain voltage level, e.g. 
$180 \mathrm{kV}$ at $50 \mathrm{~mm}$ gap, the stopping length in both oils starts to increase quickly with applied voltage having relatively larger standard deviations, which was also observed in other mineral oils under impulse voltages [11-13]. The increasing rate of negative streamers above this threshold voltage in the GTL oil (e.g. about $0.2 \mathrm{~mm} / \mathrm{kV}$ at $50 \mathrm{~mm}$ gap) is lower than that in the mineral oil (e.g. about $0.3 \mathrm{~mm} / \mathrm{kV}$ at $50 \mathrm{~mm}$ gap), which leads to a higher negative breakdown voltage in the GTL oil.

\subsection{AVERAGE PROPAGATION VELOCITY}

The average propagation velocity of negative streamers in the GTL oil and the mineral oil at both $25 \mathrm{~mm}$ and $50 \mathrm{~mm}$ gaps is shown in Figure 3. Below the breakdown voltage, the streamer average propagation velocity is calculated by using streamer propagation length divided by propagation time. Above the breakdown voltage, the streamer average propagation velocity is calculated by using gap distance divided by time to breakdown. The acceleration voltages $\mathrm{V}_{\mathrm{a}}$ are marked in Figure 3 as references.

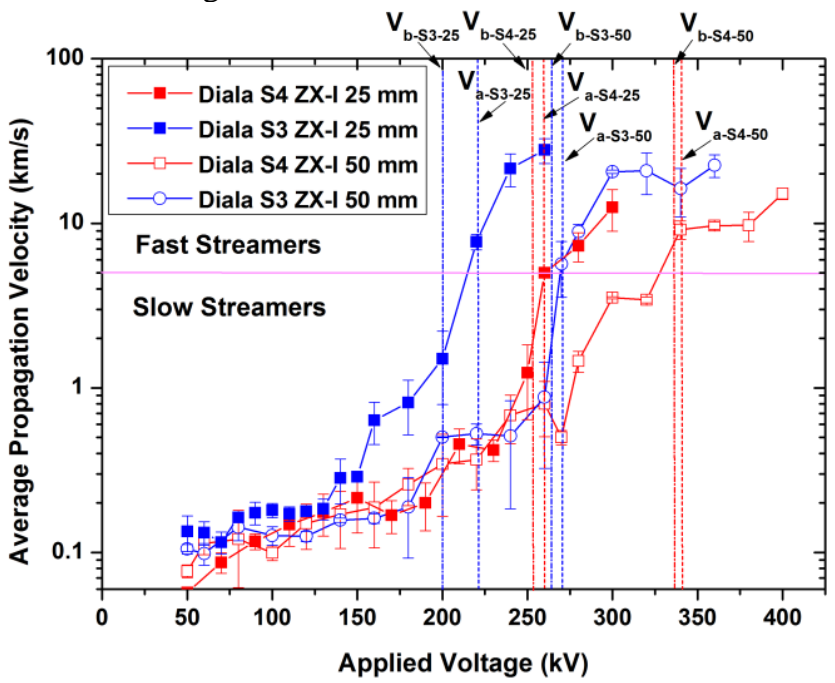

Figure 3. Streamer average propagation velocity of the GTL oil and the mineral oil, negative polarity $\left(\mathrm{V}_{\mathrm{a}-\mathrm{S} 4-\mathrm{xx}}\right.$ and $\mathrm{V}_{\mathrm{a}-\mathrm{S} 3-\mathrm{xx}}$ stand for acceleration voltage of Diala S4 ZX-I and Diala S3 ZX-I at gap distance of xx mm, respectively).

It is shown in Figure 3 that at both $25 \mathrm{~mm}$ and $50 \mathrm{~mm}$ gaps, the average propagation velocity of negative streamers increases obviously with the increase of applied voltage, but the step changes, which are usually observed under positive polarity [1], are less distinguishable under negative polarity. Below breakdown voltage $V_{b}$, the average propagation velocity of negative streamers in both oils increases gradually with applied voltage, from about $0.1 \mathrm{~km} / \mathrm{s}$ at initiation stages to about $1-4 \mathrm{~km} / \mathrm{s}$ at voltages just below $V_{b}$. There is no obvious difference in the increasing rate of the average streamer propagation velocities between the two oils at this stage. Just above $\mathrm{V}_{\mathrm{b}}$, a noticeable acceleration in streamer propagation exists where the average propagation velocity exceeds $5 \mathrm{~km} / \mathrm{s}$ (this is also used to define $\mathrm{V}_{\mathrm{a}}$ ). At the same voltage level, the average propagation velocity in the GTL oil is obviously lower than that in the mineral oil, e.g. at gap distance of $50 \mathrm{~mm}$ and applied voltage of $360 \mathrm{kV}$, the average streamer propagation velocity in the mineral oil is $22.5 \mathrm{~km} / \mathrm{s}$, which is 2.3 times higher than the average velocity in the GTL oil, $9.7 \mathrm{~km} / \mathrm{s}$. It is found that at the same voltage level, the average propagation velocity of negative streamers in both oils is much lower than that of positive streamers [1].

Table 2 summaries both the breakdown voltages and the acceleration voltages for the GTL oil and the mineral oil obtained under negative polarity. Firstly, it is found that GTL oil has a much higher breakdown voltage and acceleration voltage than the mineral oil, e.g. at $50 \mathrm{~mm}$ gap, the breakdown voltage of the GTL oil is $334.4 \mathrm{kV}$, which is 1.3 times higher than that of the mineral oil. Secondly, it is observed that the acceleration voltage for both oils is only slightly higher than the breakdown voltage.

Table 2. Breakdown voltages and acceleration voltages of the GTL oil and the mineral oil at gap distances of $25 \mathrm{~mm}$ and $50 \mathrm{~mm}$.

\begin{tabular}{ccccc}
\hline \multirow{3}{*}{$25 \mathrm{~mm}$} & Test Condition & $\mathbf{V}_{\mathbf{b}}(\mathbf{k V})$ & $\mathbf{V}_{\mathbf{a}}(\mathbf{k V})$ & $\mathbf{V}_{\mathbf{a}} / \mathbf{V}_{\mathbf{b}}$ \\
& Diala S4 ZX-I & 251.2 & 260 & 1.04 \\
& Diala S3 ZX-I & 197.0 & 220 & 1.12 \\
\multirow{3}{*}{$50 \mathrm{~mm}$} & Diala S4 ZX-I & 334.4 & 340 & 1.02 \\
& Diala S3 ZX-I & 263.3 & 270 & 1.03 \\
\hline
\end{tabular}

\subsection{STREAMER AREA}

In this paper, the streamer area is defined as the apparent area occupied by all streamer channels in the $2 \mathrm{D}$ image, which is measured by a self-developed MATLAB program.

Figure 4 shows the relationship between the streamer area and the applied voltage in both the GTL oil and the mineral oil under negative polarity. The area of slow streamers in both oils increases gradually with applied voltage till $\mathrm{V}_{\mathrm{a}}$ is reached, where the streamer area in both testing oils reaches the peak value. This suggests that the negative slow streamer is most branched at this voltage, as shown in $\mathrm{C} 1$ and $\mathrm{C} 2$ in Figure $4 \mathrm{~b}$. Then there is a sudden drop in the streamer area just above $\mathrm{V}_{\mathrm{a}}$, but it starts to increase again when the applied voltage is further increased. This suggests that negative fast streamers also become more branched in both testing oils with the increase of applied voltage. To sum up, the shape evolution of both fast streamers and slow streamers follow the same relationship with applied voltage: the higher the voltage is applied, the larger area a streamer has. Similar relationships were reported in a synthetic ester under negative 1.2/50 $\mu \mathrm{s}$ lightning impulse voltages [11] and in a paraffinic model oil under negative $0.5 / 1700 \mu$ s step impulse voltages [13].

From $\mathrm{V}_{\mathrm{b}}$ to $\mathrm{V}_{\mathrm{a}}$, as shown in $\mathrm{C} 1$ and $\mathrm{C} 2$ in Figure $4 \mathrm{~b}$, the branches of negative streamers are much less than those of positive streamers observed in [1]. There is hardly any shielding effect between the branches of negative streamers, so the acceleration voltage is only slightly higher than the breakdown voltage under negative polarity.

Below $\mathrm{V}_{\mathrm{b}}$, at the same voltage level, negative streamers in the mineral oil (as shown in A2 and B2 in Figure 4b) propagate further and have more offshoots compared to those in the GTL oil (as shown in A1 and B1 in Figure 4b). Negative streamers are easier to propagate in the mineral oil, resulting in a lower breakdown voltage than that in the GTL oil.

It is generally observed that molecules with an electron scavenging function facilitate the negative streamer propagation [2, 4-8, 12, 13]. A previous publication [13] 
indicated that both negative breakdown voltage and acceleration voltage of a paraffinic model oil were reduced by adding electron scavenger additives. Relating this to the present results, it indicates that the GTL oil has less components with an electron scavenging function than the mineral oil. This is confirmed by the fact that the GTL oil almost does not have aromatic components with electron scavenger functions.

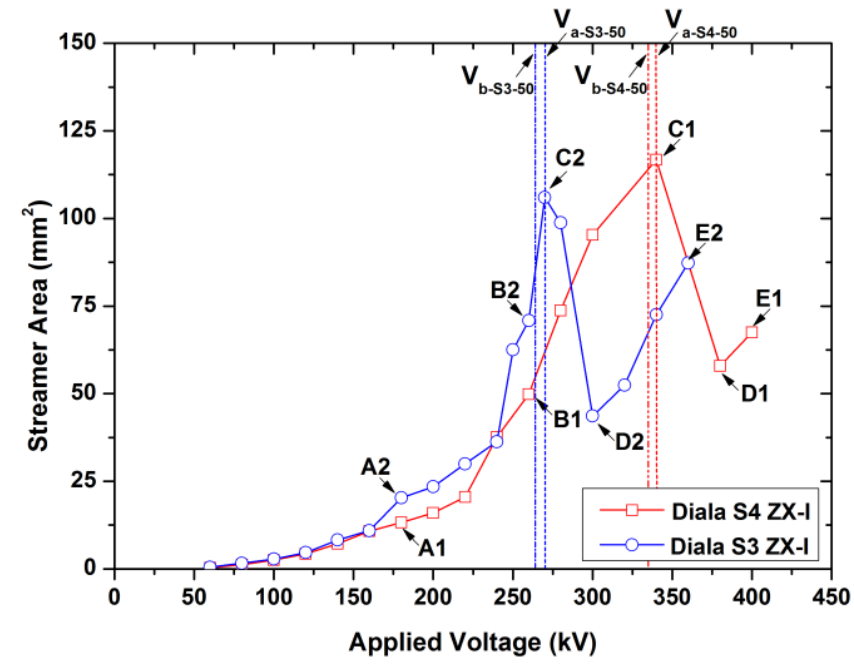

(a) Streamer area versus applied voltage

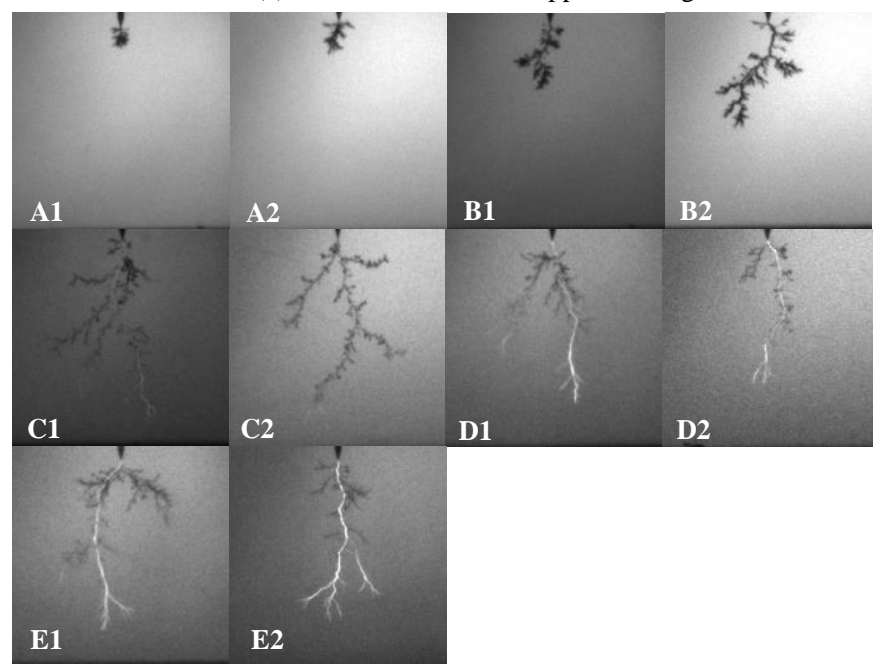

(b) Correlated streamer shapes at different stages in (a)

Figure 4. Evolution of streamer shapes along applied voltage for the GTL oil (A1-E1) and the mineral oil (A2-E2), negative polarity, $\mathrm{d}=50 \mathrm{~mm}$; the ground electrode is at the bottom edge of the streamer images.

\section{STREAMER PROPAGATION MECHANISMS WITH FOCUS ON FAST STREAMER PROPAGATION}

\subsection{PROPAGATION MODE FOR NEGATIVE STREAMERS}

In order to distinguish the evolution of detailed streamer propagation process in both oils, a velocity based streamer mode classification was used in this study. Negative streamers with different instantaneous velocities (calculated from imaging frame sequences) during propagation are classified into 4 modes: the $1^{\text {st }}$ mode streamers propagate with velocity of below $0.5 \mathrm{~km} / \mathrm{s}$, whereas the $2^{\text {nd }}$ mode streamers propagate faster with typical velocity from $0.5 \mathrm{~km} / \mathrm{s}$ to $4 \mathrm{~km} / \mathrm{s}$. The $3^{\text {rd }}$ mode streamers with a typical velocity of $5-8 \mathrm{~km} / \mathrm{s}$ and the $4^{\text {th }}$ mode streamers with a typical velocity higher than $10 \mathrm{~km} / \mathrm{s}$ are defined as fast streamers, which can be observed above the acceleration voltage. Similar velocity based classifications for the propagation modes of negative streamers were used in [12, $21,22]$.

A cross-comparison of the evolution of streamer mode with applied voltage in the GTL oil and the mineral oil under negative polarity is shown in Figure 5. It was found that similar evolution of streamer mode was observed at both $25 \mathrm{~mm}$ and $50 \mathrm{~mm}$ gaps, and only the results obtained at $50 \mathrm{~mm}$ gap were discussed in detail in this section.

It is shown in Figure 5 that in both oils, with the increase of applied voltage, the propagation of negative streamers changes gradually from slow modes $\left(1^{\text {st }}\right.$ mode and $2^{\text {nd }}$ mode) below $V_{b}$ to fast modes $\left(4^{\text {th }}+3^{\text {rd }}\right.$ mode and $4^{\text {th }}$ mode $)$ above $V_{a}$. The threshold voltage for the transition from slow $2^{\text {nd }}$ mode to fast $4^{\text {th }}+3^{\text {rd }}$ mode is obviously higher in the GTL oil, which indicates that the GTL oil has a higher resistance to negative fast streamers compared to the mineral oil.

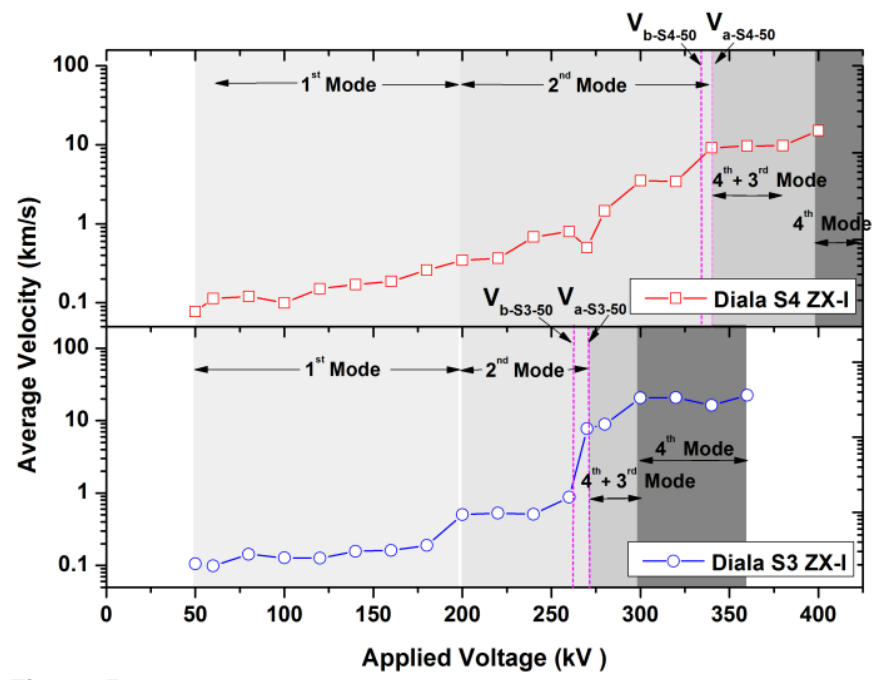

Figure 5. Streamer propagation mode versus applied voltage in the GTL oil and the mineral oil; $\mathrm{d}=50 \mathrm{~mm}$.

Examples of detailed propagation progress of negative streamers are described with shadowgraph images on a frame by frame basis. As shown in Figure 6, when the applied voltages are well below $\mathrm{V}_{\mathrm{b}}$, negative streamers in both oils propagate in the $1^{\text {st }}$ mode. The $1^{\text {st }}$ mode streamer has a bushlike shape, consisting of multiple dense short branches. The streamers in this mode have a very low velocity of 0.1 $0.3 \mathrm{~km} / \mathrm{s}$ and they can only propagate for a very short distance of less than $8 \mathrm{~mm}$. The $1^{\text {st }}$ mode negative streamers with similar characteristics were also observed in $[12,13]$ when the applied voltage is very low.

At higher voltages up to $\mathrm{V}_{\mathrm{a}}$, the $2^{\text {nd }}$ mode negative streamers can be observed, as shown in Figure 7 . The $2^{\text {nd }}$ mode streamers are more filamentary than the $1^{\text {st }}$ mode streamers. The $2^{\text {nd }}$ mode streamers generally have a tree-like shape, consisting of a few main branches with small offshoots surrounded. 
Compared to positive $2^{\text {nd }}$ mode streamers observed in [1], negative $2^{\text {nd }}$ mode streamers have fewer main branches and the offshoots do not propagate obviously after the appearance. The branches of the negative $2^{\text {nd }}$ mode streamers are thicker than those of the positive $2^{\text {nd }}$ mode streamers. In addition, there is no significant increase in the number of streamer branches of the negative $2^{\text {nd }}$ mode streamers when the applied voltage is further increased.

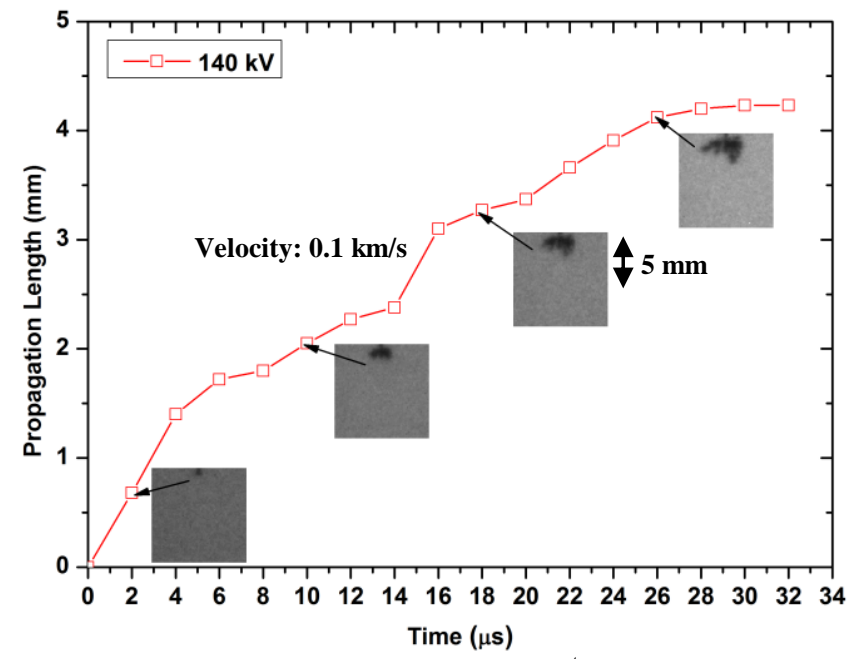

Figure 6. The typical propagation processes of $1^{\text {st }}$ mode negative streamers at low voltages below $\mathrm{V}_{\mathrm{b}}$; GTL oil, $\mathrm{d}=50 \mathrm{~mm}$.

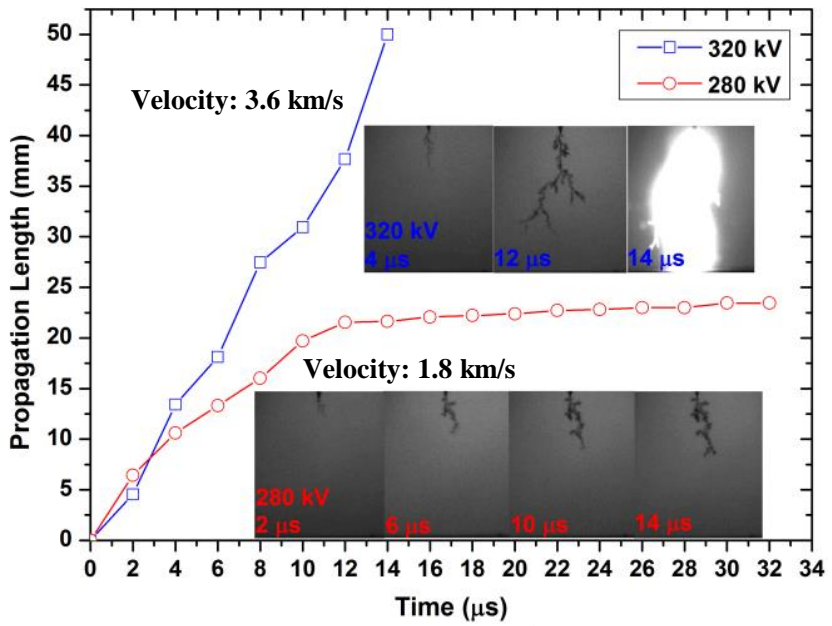

Figure 7. The typical propagation process of $2^{\text {nd }}$ mode negative streamers in the GTL oil, d=50 mm; the ground electrode is at the bottom edge of the streamer images.

As shown in Figure 8, when $\mathrm{V}_{\mathrm{a}}$ is reached, the propagation of negative streamers in both oils switches to the fast $4^{\text {th }}+3^{\text {rd }}$ mode. Concerning the detailed process, the propagation of $4^{\text {th }}+3^{\text {rd }}$ mode negative streamers is indeed a 3 -stage process, i.e. initial stage, intermediate stage and final stage. In the initial stage, the streamer propagation starts with the formation of a couple of primary bright channels. These bright channels have a filamentary shape and propagate in the $4^{\text {th }}$ mode with a typical velocity of over $10 \mathrm{~km} / \mathrm{s}$. However these bright channels can only propagate for a short period (less than 0.5 $\mu \mathrm{s})$. After this period, the intermediate stage of streamer propagation begins. At this stage, a few dark channels emanate from the primary bright channels. These dark channels propagate in the $3^{\text {rd }}$ mode with a typical velocity of $5-8 \mathrm{~km} / \mathrm{s}$. The shape of these $3^{\text {rd }}$ mode dark channels is similar to that of the $2^{\text {nd }}$ mode streamer observed at lower voltage, but has obviously more branches. Eventually, when the furthest dark branch gets close enough to the plane electrode, it suddenly turns to a bright channel and triggers the final stage of the streamer propagation. This main bright channel propagates in the $4^{\text {th }}$ mode with a typical velocity of over $10 \mathrm{~km} / \mathrm{s}$ toward the ground electrode and causes the breakdown. During the propagation of the main bright channel, a lot of small offshoots also appear along the bright channel.

This combination mode consisting of $3^{\text {rd }}$ and $4^{\text {th }}$ mode streamers above the acceleration voltage was also mentioned in a paraffinic model oil under negative $0.5 / 1700 \mu \mathrm{s}$ step impulse voltages $[12,13]$.

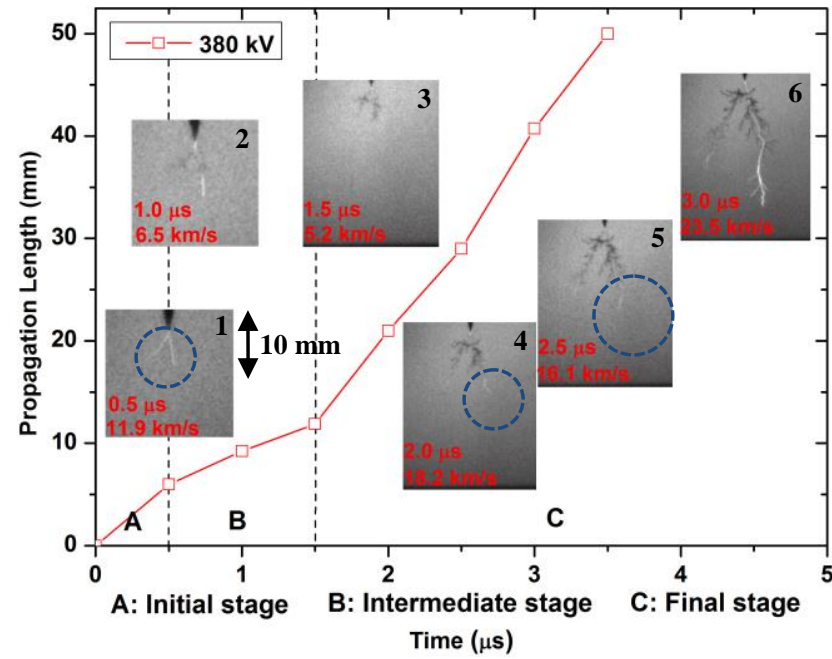

Figure 8 The typical propagation process of $4^{\text {th }}+3^{\text {rd }}$ mode negative streamers in the GTL oil, $d=50 \mathrm{~mm}$; the ground electrode is at the bottom edge of the streamer images from frame 3 to frame 6.

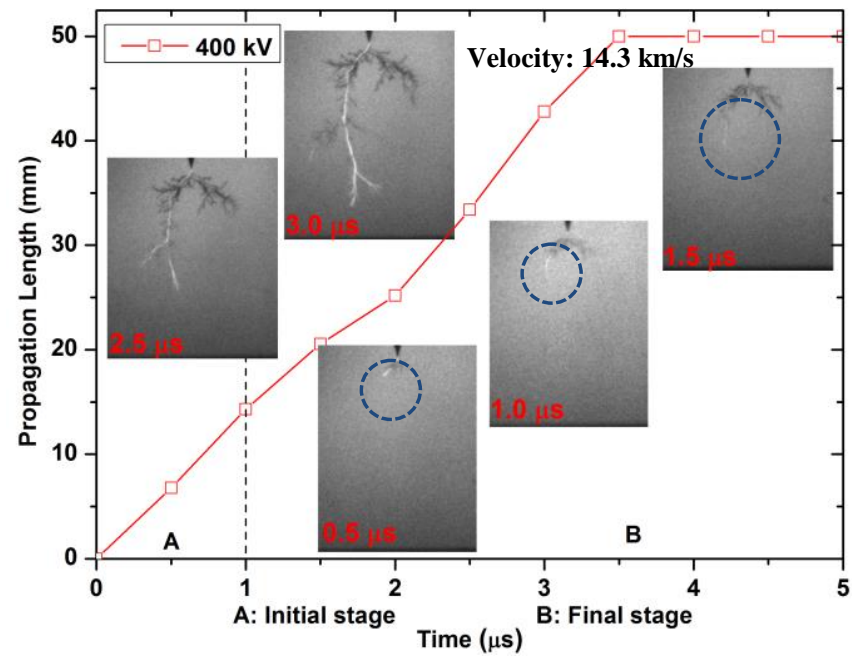

Figure 9 The typical propagation process of $4^{\text {th }}$ mode negative streamers in the GTL oil, d=50 mm; the ground electrode is at the bottom edge of the streamer images.

When the applied voltage is further increased, the propagation of negative streamers in both oils switches to the $4^{\text {th }}$ mode with a constant velocity of over $10 \mathrm{~km} / \mathrm{s}$, as shown in Figure 9. Once the primary bright channels are formed in 
initial stage, instead of quickly die-out in previous $4^{\text {th }}+3^{\text {rd }}$ combination mode, they will continuously propagate towards the plane electrode and lead to breakdown, i.e. the propagation directly switches from initial stage into the final stage. In the final stage, a few dark branches with velocity in the $3^{\text {rd }}$ mode could appear simultaneously during the propagation of the main bright channels.

\subsection{EVOLUTION OF STREAMER CHARACTERISTICS ABOVE ACCELERATION VOLTAGE}

As described in section 4.1, above the acceleration voltage, negative streamers in both oils can propagate either as $4^{\text {th }}$ mode bright channels or as $3^{\text {rd }}$ mode dark channels at different propagation stages. The changing trend of streamer velocity with the increase of applied voltage at different propagation stages is shown in Figure 10. In the initial stage, the propagation velocity of the primary bright channels in the mineral oil increases gradually from approximately $10 \mathrm{~km} / \mathrm{s}$ to over $20 \mathrm{~km} / \mathrm{s}$ with the increase of applied voltage, whereas the propagation velocity in the GTL oil does not change obviously within the investigated voltage range. Overall the propagation velocity of the primary bright channels in the GTL oil is lower than that in the mineral oil. In the intermediate stage, the propagation velocity of the dark channels is around $6 \mathrm{~km} / \mathrm{s}$ and in the final stage, the propagation velocity of the bright channels lies between $15 \mathrm{~km} / \mathrm{s}$ and $25 \mathrm{~km} / \mathrm{s}$. It is noticed that in the intermediate stage and the final stage, the propagation velocity of main streamer channels does not change with the increase of applied voltage and there is not a big difference in the streamer propagation velocity between the GTL oil and the mineral oil.

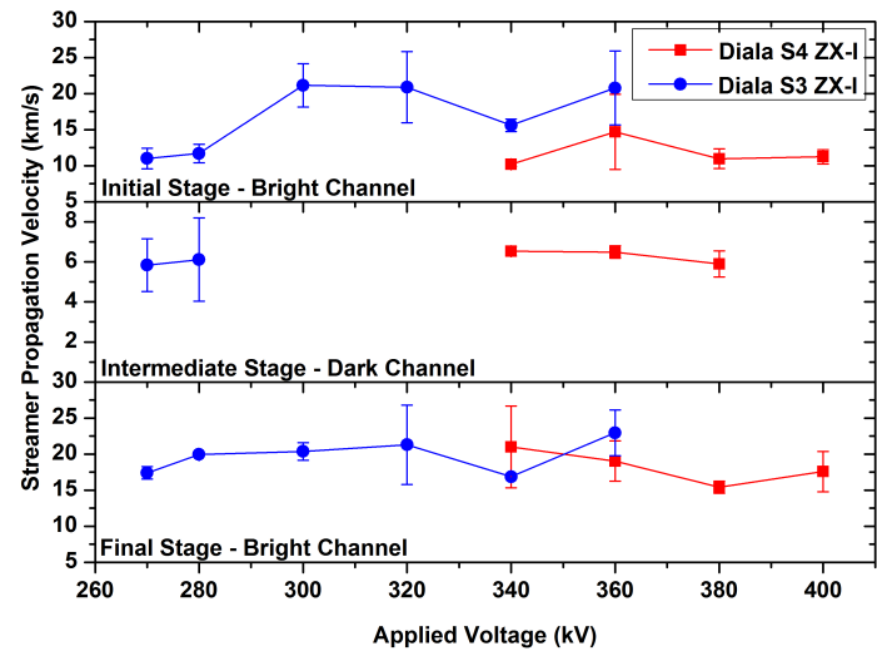

Figure 10 Average propagation velocity versus applied voltage for bright channels and dark channels at different propagation stages; $\mathrm{d}=50 \mathrm{~mm}$.

Furthermore, a closer look at the development of streamer areas in both oils at voltage levels above $\mathrm{V}_{\mathrm{a}}$ is shown in Figure 11. The same as the analyses conducted under positive polarity [1], the streamer areas are split into the area of dark channels and bright channels and the applied voltages are normalised to the ratio of acceleration voltage. It is shown in Figure 11a that above $\mathrm{V}_{\mathrm{a}}$, with the increase of applied voltage, the area of bright channels in both oils increases gradually and the area of dark channels in both oils tends to reduce gradually. The changing rate of areas of bright channels and dark channels in the mineral oil is slightly higher than that in the GTL oil. It is indicated that above acceleration voltage, the development of fast streamers in both oils is the consequence of transition from dark streamer channels to bright streamer channels. The mineral oil favors this transition process more than the GTL oil. Relating this finding to the results presented in Figure 10, the faster transition from dark channels to bright channels for the mineral oil could be due to the more efficient propagation of primary bright channels in the initial stage.

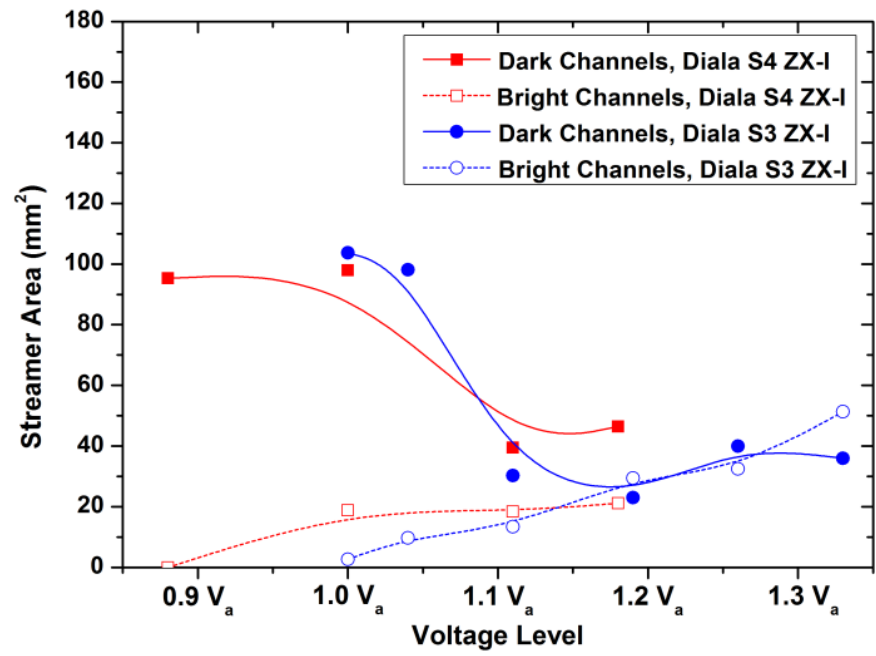

(a) Areas of the dark channels and the bright channels versus voltage level
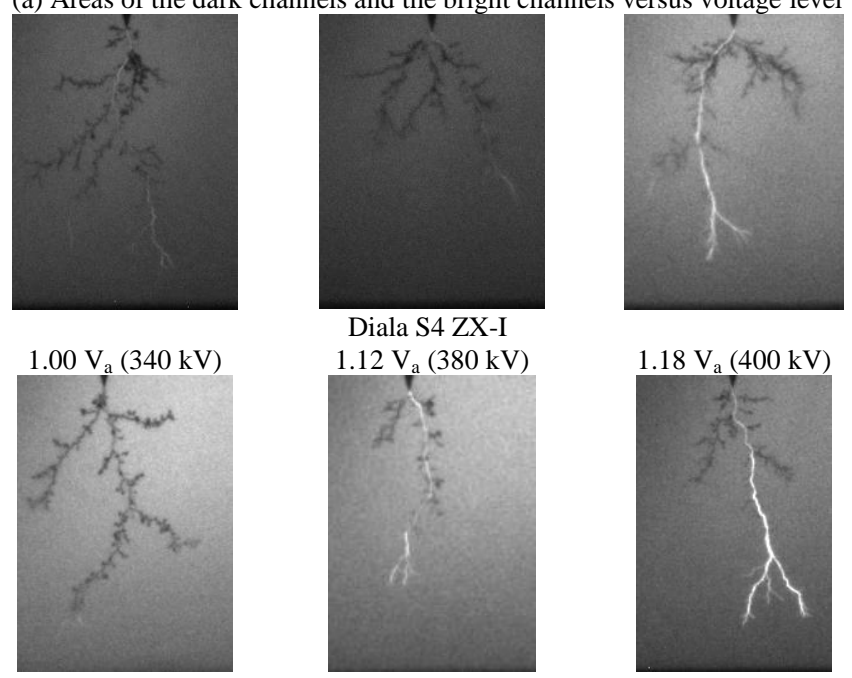

$1.00 \mathrm{~V}_{\mathrm{a}}(270 \mathrm{kV})$

Diala S4 ZX-I

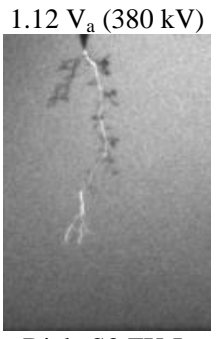

Diala S3 ZX-I

$1.11 \mathrm{~V}_{\mathrm{a}}(300 \mathrm{kV})$

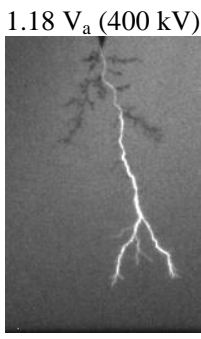

$1.19 \mathrm{~V}_{\mathrm{a}}(320 \mathrm{kV})$

(b) Streamer shapes in both testing liquids under different voltage levels Figure 11. Shape changes of $4^{\text {th }}+3^{\text {rd }}$ streamers above acceleration voltage, $\mathrm{d}=50 \mathrm{~mm}$; the ground electrode is at the bottom edge of the streamer images.

In addition, comparing with the development of the area of bright channels and dark channels under positive polarity [1], it is found that the changing rate for the transition from dark channels to bright channels in both oils is slower under negative polarity. Accordingly, above acceleration voltage, the average velocity of positive streamers in both oils of 15-120 $\mathrm{km} / \mathrm{s}$ is much higher than that of negative streamers of 8 $20 \mathrm{~km} / \mathrm{s}$. 


\subsection{FAST NEGATIVE STREAMERS VERSUS FAST POSITIVE STREAMERS: DIFFERENCE IN PROPAGATION MECHANISMS}

It is indicated in the previous paper [1] that for the propagation of fast streamers, a charge splitting process at the streamer tip is considered to be responsible for the propagation of dark channels, whereas a charge amplification process at the streamer tip is responsible for the propagation of bright channels. In addition, research in [12, 13, 15, 23-27] suggested that the electronic process at the streamer tips, i.e. electron avalanche, plays an important role in the propagation of fast negative streamers. Based on these hypotheses, a model for describing the propagation mechanism of fast negative streamers in the GTL oil and the mineral oil is proposed, as shown in Figure 12. The model proposed in the previous paper [1] for describing the propagation mechanism of fast postive streamers is also presented for comparison.

Stage 1 in Figure 12 correspnds to the initial stage of fast streamer propagation. Under negative polarity, fast streamers propagate as bright channels in the initial stage, whereas fast streamers under positive polarity propagate as dark channels in the initial stage. The propagation mechanism related to this difference can be explained as follows: under negative polarity, electrons are continuously injected from the point electrode into the surrounding oils. These electrons are trapped at a short distance from the point electrode and lead to a charge amplification in front of the electrode. When the applied voltage is sufficiently high, i.e. the acceleration voltage is reached, the concentration of the accumulated charges in front of the point electrode becomes high enough and lead to the ionization of the surrounding main naphthenic/paraffinic molecules. Ionization of these main molecules with a high ionization potential leads to a high field enhancement in front of the point electrode. This high field allows more efficient field ionization of a large amount of these main molecules. As a consequence, a bright channel with high conductivity emanates from the point electrode.

For fast postive streamers in the initial stage, as already explained in [1], when a high voltage is applied, there is no electron injection from the point electrode, charge amplication does not occur and only easily ionizable molecules, e.g. aromatic molecules, can be ionized and the initial dark channel is formed. This initial dark channel has low conductivity and charge splitting happens in the branching process. Therefore we can only see dark channels in the initial stage under positive polarity.

Stage 2 in Figure 12 corresponds to the intermediate stage of fast streamer propagation. Under negative polarity, the moving direction of the seed electrons accumulated at the streamer tip is toward the far end of the ionization zone. When the primary bright channel is propagating, the charge input/seed electrons at the streamer tip tend to split into a large area in front of the streamer tip. Therefore after propagating for a short distance, the propagation of negative streamers becomes less efficient due to the distortion of charge and field at the streamer tip. Then only easily ionizable molecules can be ionized and the dark channels are formed. Once the propagation of fast negative streamers turns to dark channels, the charge splitting process at the streamer tips continuously occurs and becomes dominant, therefore a lot of small dark channels appear along the streamer main branches.

For fast postive streamers in intermediate stage, as explained in [1], either charge amplification or charge splitting could occur at streamer tips depending on whether streamer tips could accumulate charges faster than they are neutralized or not during streamer propagating/branching. Therefore both dark streamer channels and bright streamer channels exist in intermediate stage.

Stage 3 in Figure 12 corresponds to the final stage of fast streamer propagation. Under negative polarity, at the end of intermediate stage, when the furthest dark channel approaches the ground electrode, there is a chance that the local electrical field around one of the streamer tips can be enhanced due to the reduction of distance between the streamer tip and the ground electrode. Under this high local field, electrons at the streamer tips are accelerated and the possible more efficient ionization processes, e.g. photo-ionization and molecular ionization [25-27] could occur. As a result, charge density is amplified at the streamer tip and the surrounding main naphthenic/ paraffinic molecules are ionized. This streamer tip becomes highly conductive and turns to a bright channel. At the same time, the impulse voltage is still around the peak value, therefore the tip field and the charge density of the bright channels can be maintained to continuously ionize the main naphthenic/paraffinic molecules in front of the bright channels. As a consequence, the bright channels continually propagate toward the ground electrode and cause breakdown.

For fast postive streamers in final stage, as explained in [1], the impulse voltage is rising or around the peak value, the tip field and the charge density of the bright channels can be maintained to continuously ionize the main naphthenic/paraffinic molecules in front of the parent bright channels formed in previous propagation stages. Therefore bright channels continuously propagate toward the ground electrode and cause breakdown.

The model shown in Figure 12 can be used to explain the differences in streamer characteristics of fast negative streamers between the GTL oil and the mineral oil. It is confirmed that the mineral oil has more aromatic components compared to the GTL oil. In the initial stage, the electron scavenging/trapping properties of these aromatic components can help to confine the seed electrons at the streamer tip, and hence raise the tip field facilitating the propagation of primary bright channels. Therefore the primary bright channels in the mineral oil propagate with a higher propagation velocity compared to those in the GTL oil, as shown in Figure 10. The more efficient propagation in initial stage explains the higher velocity of the fast negative streamers in the mineral oil than that in the GTL oil shown in Figure 3.

Research in [12, 29, 30] suggested that both electronic and gaseous processes at streamer tips are responsible for the development of fast streamers. In addition, streamer propagation models shown in Figure 12 indicated that charge splitting process is dominant for the branching of dark 
channels, whereas charge amplification process is dominant for the branching of bright channels. Based on these conclusions, physical nature of dark channels and bright channels are discussed, as show in Figure 13. The development of dark channels is possibly controlled by gaseous processes occurred at streamer tips, i.e. liquid superheating introduced by electron avalanche, and the following shockwave emissions as well as promotion of gas/liquid interfaces. Electronic processes play less important roles in the branching of dark channels. Dark channels are possibly gaseous channels with relatively low conductivity. On the other hand, the branching of bright channels is possibly dominated by the electronic processes occurred at streamer tips, such as photo ionizations and molecular ionizations introduced by charge injections and large electron avalanches.

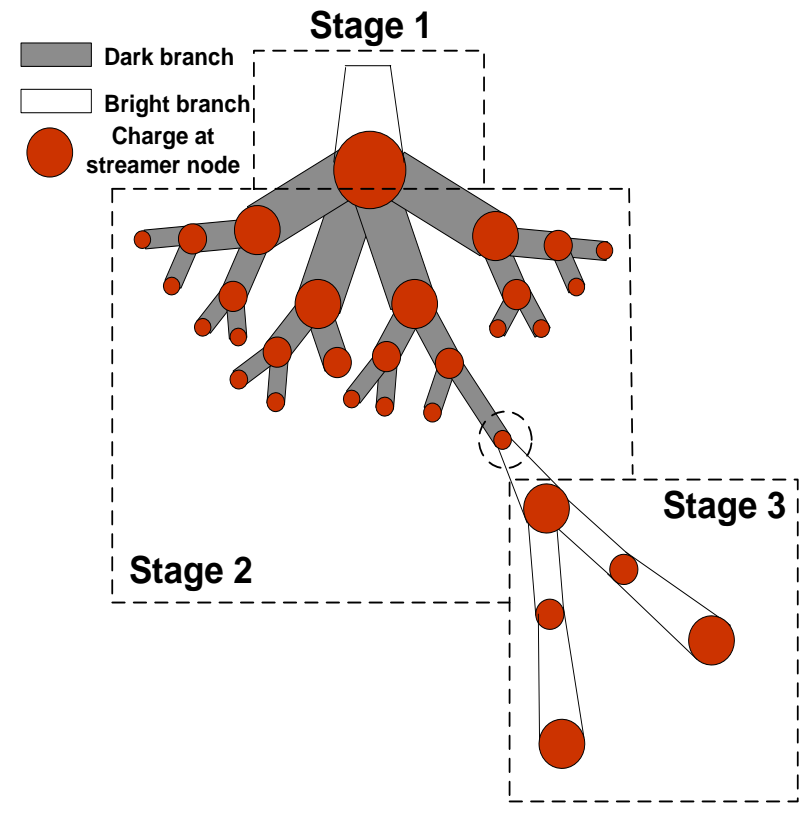

Negative Polarity
Gaseous processes have less effects on the development of bright channels. Bright channels are possibly highly conductive plasma channels.

In order to confirm the physical nature of dark channels and bright channels of fast streamers, parameters for describing the electronic processes such as the electric field stress and energy dissipation at streamer tips, as well as parameters for describing the gaseous processes such as shockwave propagation velocity and pressure profile inside streamer channels need to be quantitatively estimated. By figuring out the dominant process at different propagation stages, the model based on Figure 12, can be further improved for describing the transition of streamer propagation modes.

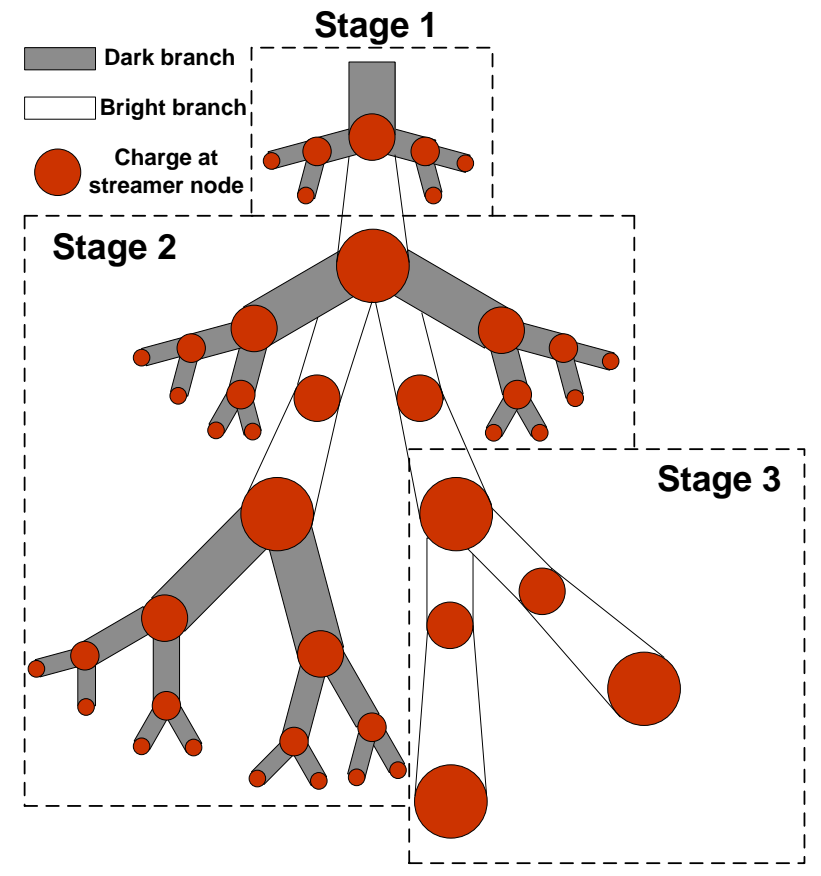

Positive Polarity

Figure 12. Propagation models for fast streamers in the GTL oil and the mineral oil under both negative and positive polarities

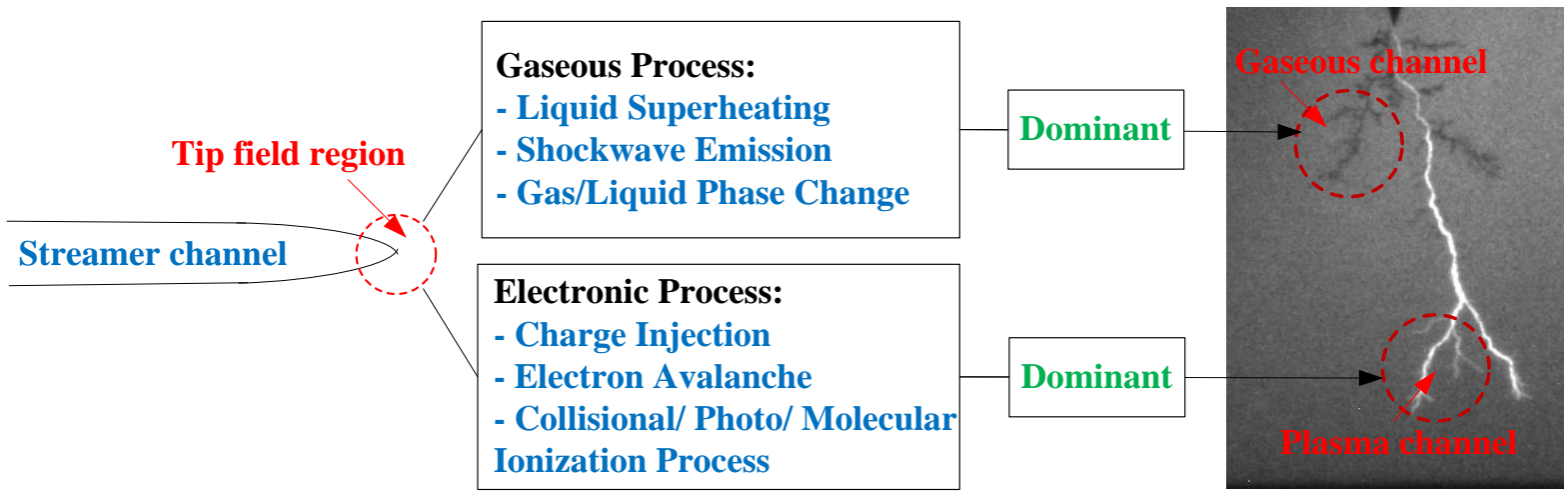

Figure 13 The relationship between microcosmic processes occurred at streamer tips and physical nature of streamer channels

\section{CONCLUSIONS}

The streamer and breakdown mechanisms of a GTL oil Diala S4 ZX-I and a mineral oil Diala S3 ZX-I were investigated under negative lightning impulse voltages at 25 $\mathrm{mm}$ and $50 \mathrm{~mm}$ point-plane gaps. The results showed that at the same voltage level, non-breakdown streamers in the GTL oil have shorter stopping length compared to those in the 
mineral oil, resulting in higher breakdown voltages of the GTL oil under negative polarity. Further studies of streamer average velocity showed that under negative polarity, the GTL oil has a higher acceleration voltage than the mineral oil. This is most likely due to the fact that the GTL oil has a much lower aromatic content with electron scavenging property compared to the mineral oil.

The study of streamer propagation mode showed that above acceleration voltage, the propagation of fast negative streamers in both oils is a 3 -stage process. The $4^{\text {th }}$ mode streamer starts as a primary bright channel in the initial stage and then the $3^{\text {rd }}$ mode streamer appears as dark channels in the intermediate stage. In the final stage, the furthest $3^{\text {rd }}$ mode dark channel switches to the $4^{\text {th }}$ mode bright channel when it gets close enough to the ground electrode and this will quickly leads to breakdown.

Above the acceleration voltage, fast negative streamers always initiate as bright channels in the initial stage, whereas fast positive streamers always initiate in the form of dark channels. It is also found that bright channels of fast positive streamers are more luminous and propagate much faster compared to those of fast negative streamers, which could be caused by more efficient charge amplification at the streamer tip. Discussions on formation mechanism of streamers indicated that dark channels are possible low conductive gaseous channels, whereas bright channels are highly conductive plasma channels.

\section{ACKNOWLEDGMENT}

The authors would like to thank Shell for providing the testing oils. The authors also would like to acknowledge the use made of Specialised Imaging SIM 16 Camera which was borrowed from the EPSRC (Engineering and Physical Sciences Research Council) Engineering Instrument Pool.

\section{REFERENCES}

[1] W. Lu and Q. Liu, "Pre-breakdown and breakdown phenomena of an inhibited Gas-To-Liquid (GTL) hydrocarbon transformer oil under positve lightning impulse voltage", IEEE Trans. Dielectr. Electri. Insul., vol. 23, No. 4, pp. 2450-2461, 2016.

[2] J. C. Devins, S. J. Rzad, J. Robert, and A. J. Schwabe, "Breakdown and pre-breakdown phenomena in liquids," J. Appl. Phys., Vol. 52 No. 7, pp. 4531-4545, 1981.

[3] A. Beroual, M. Zhan, A. Badent and K. Kist, "Propagation and structure of streamers in liquid dielectrics," IEEE Electr. Insul. Mag., Vol. 14, No. 2, pp. 6-17, 1998.

[4] G. J. FitzPatrick, P. J. McKenny, and E. O. Forster, "The effect of pressure on streamer inception and propagation in liquid hydrocarbons," IEEE Trans. Dielectr. Electri. Insul., vol. 25, No. 4, pp. 672-682, 1990.

[5] Y. Nakao, H. Itoh, S. Hoshino, Y. Sakai, and H. Tagashira, "Effects of additives on prebreakdown phenomena in n-hexane," IEEE Trans. Dielectr. Electri. Insul., vol. 1, No. 3, pp. 383-389, 1994.

[6] D. Linhjell, S. Ingebrigtsen, L. E. Lundgaard, and M. Unge, "Streamer in long point-plane gaps in cyclohexane with and without additives under step voltage," IEEE Int'l. Conf. Dielectr. Liquids (ICDL), Trondheim, Norway, pp. 1-5, 2011.

[7] S. Ingebrigtsen, L.E. Lundgaard, and P.O. Astrand, "Effects of additives on prebreakdown phenomena in liquid cyclohexane: I. Streamer initiation”, J. Phys. D: Appl. Phys., Vol. 40, pp. 5161-5169, 2007.

[8] S. Ingebrigtsen, L. E. Lundgaard and P. O. Astrand, "Effects of additives on prebreakdown phenomena in liquid cyclohexane: II. Streamer propagation," J. Phys. D: Appl. Phys., Vol. 40, No. 18, pp. 5624-5634, 2007.
[9] Q. Liu, Z. D. Wang, and O. Lesaint, "Comparison of streamer propagation in mineral oils under lightning and step impulse voltages," IEEE Int'l. Conf. Dielectr. Liquids (ICDL), Bled, Slovenia, Bled, Slovenia, pp. 1-4, 2014.

[10] Q. Liu and Z. D. Wang, "Streamer characteristic and breakdown in synthetic and natural ester transformer liquids with pressboard interface under lightning impulse voltage," IEEE Trans. Dielectr. Electri. Insul., Vol. 18, No. 6, pp. 1908-1917, 2011.

[11] Q. Liu and Z. D. Wang, "Streamer characteristic and breakdown in synthetic and natural ester transformer liquids under standard lightning impulse voltage," IEEE Trans. Dielectr. Electri. Insul., Vol. 18, No. 1, pp. 285-294, 2011.

[12] N. V. Dung and L. E. Lundgaard, "Effects of reduced pressure and additives on streamers in white oil in long point-plane gap," J. Appl. Phys., vol. 46, 2013.

[13] N. V. Dung, H. K. Hoidalen, D. Linhjell, L. E. Lundgaard, and M. Unge, "Influence of impurities and additives on negative streamers in paraffinic model oil," IEEE Trans. Dielectr. Electri. Insul., Vol. 20, pp. 876-886, 2013.

[14] P. Rozga, "Streamer propagation in small gaps of synthetic ester and mineral oil under lightning impulse," IEEE Trans. Dielectr. Electri. Insul., Vol. 22, No. 5, pp. 2754-2762, 2015.

[15] G. Massala and O. Lesaint, "A comparision of negative and positive streamers in mineral oil at large gaps," J. Appl. Phys., vol. 34, pp. 15251532, 2001.

[16] P. Smith, "The benefits of inhibited transformer oils using the latest gasto-liquid based technology," Euro Techcon, Glasgow, UK, pp.1-8, 2013.

[17] W. Lu, Q. Liu, Z. D. Wang, and P. W. R. Smith, "Lightning impulse breakdown performance of an inhibited Gas-To-Liquid (GTL) hydrocarbon transformer oil," IEEE Int'l. Conf. Dielectr. Liquids (ICDL), Bled, Slovenia, pp. 1-4, 2014.

[18] Z. Liu, Q. Liu, Z.D. Wang, P. Jarman, C. Krause, P.W.R. Smith, and A. Gyore, "Partial discharge behaviour of transformer liquids and the influence of moisture content," IEEE Int'l. Conf. Dielectr. Liquids (ICDL), Bled, Slovenia., pp. 1-4, 2014.

[19] S. Y. Matharage, Q. Liu and Z. D. Wang, "Ageing Assessment of kraft paper insulation through methanol in oil measurement," IEEE Trans. Dielectr. Electri. Insul., Vol. 23, No. 3, pp. 1589-1596, 2016.

[20] IEC 60897, "Methods for the determination of the lightning impulse breakdown voltage of insulating liquids," International Electrotechnical Commission, 1987.

[21] Q. Liu and Z. D. Wang, "Secondary reverse streamer observed in an ester insulating liquid under negative impulse voltage," J. Phys. D: Appl. Phys., Vol. 44, No. 40, pp. 405203(1)-405203(9), 2011.

[22] R. E. Hebner, "The liquid state and its electrical properties," NATO ASI Series, vol. B193, p. Plenum Press, 1989.

[23] P. Ceccato, "Filamentary Plasma Discharge inside Water: Initiation and Propagation of a Plasma in a Dense Medium," PhD Thesis, LPP Ecole Polytechnique, France, 2009.

[24] M. Haidara and A. Denat, "Electron multiplication in liquid yclohexane and propane," IEEE Electrical Insulation Magazine, vol. 26, pp. 592597, 1991.

[25] L.E. Lundgaard, " Propagation of positive and negative streamers in oil with and without pressboard interfaces," IEEE Trans. Dielectr. Electri. Insul., vol. 5, pp. 388-395, 1998.

[26] J. G. Hwang, M. Zahn and R. Liu, "Modelling streamers in transformer oil: the transitional fast 3rd mode streamer," [C], IEEE Conf. Properties. Applications. Dielectr. Materials (ICPADM), Harbin, China, pp. 573578, 2009.

[27] J. Jadidian M. Zahn and K. Borg, "Stochastic and deterministic causes of streamer branching in liquid dielectrics," [J], J: Appl. Phys., Vol. 114, No. 6, pp. 063301(1)-063301(11), 2013.

[28] D. Linhjell, L. Lundgaard, and G. Berg, "Streamer/Leaders from a metallic particle between parallel plane electrodes in transformer oil," IEEE Trans. Dielectr. Electri. Insul., vol. 8, pp. 1054-1063, 2001.

[29] O. Lesaint, "Pre-breakdown phenomena in liquids: propagation 'modes' and basic physical properties," [J], J. Phys. D: Appl. Phys., Vol. 49, No. 40, pp. 14401(1)-14401(22), 2016.

[30] A. Sun, C. Huo and J. Zhuang, "Formation mechanism of streamer discharges in liquids: a review," [J], IET High. Voltage., Vol. 1, No. 2, pp. 74-88, 2016. 


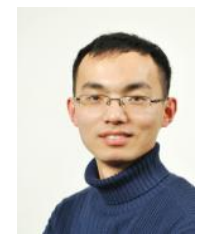

Wu Lu (S'12-M'16) obtained the B.Eng. degree in electrical and electronic engineering (2011) and the Ph.D. degree in electrical power engineering (2015) from The University of Manchester in UK. During 2015-2016 he was a Research Associate at the Power and Energy Division in the School of Electrical and Electronic Engineering at The University of Manchester. Currently he is a Lecturer in the College of Electrical Power at Shanghai University of Electric Power. His research interest is the pre-breakdown (streamer) mechanism study of alternative transformer liquids under impulse stresses.

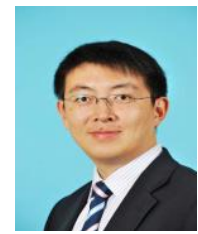

Qiang Liu (S'08-M'12) obtained the B.Eng. degree in electrical engineering (2005) and the M.Eng. degree in high voltage and electrical insulation (2008) from Xi'an Jiaotong University (XJTU) in China, and the Ph.D. degree in electrical power engineering (2011) from The University of Manchester in UK. Currently he is a Senior
Lecturer at the Power and Energy Division in the School of Electrical and Electronic Engineering at The University of Manchester. His research interests are on pre-breakdown and breakdown phenomena in liquids, alternatve transformer liquids, ageing of insulating materials, transformer thermal modelling, transformer asset management and high voltage testing.

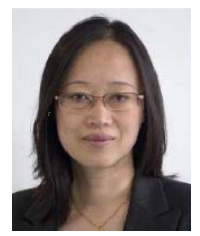

Zhongdong Wang received the B.Eng. and the M.Eng. degrees in high voltage engineering from Tsinghua University of Beijing in 1991 and 1993, respectively and the Ph.D. degree in electrical engineering from UMIST in 1999. Currently she is a Professor of High Voltage Engineering at the Power and Energy Division of the School of Electrical and Electronic Engineering at The University of Manchester. Her current research interests include condition monitoring, transformer modeling and FRA \& transients' simulation, insulation aging and alternative insulation materials for transformers. 